\title{
Aulas de Campo Ubíquas
}

\section{Edgar Marçal, Rossana M. C. Andrade, Windson Viana}

Mestrado e Doutorado em Ciência da Computação - Universidade Federal do Ceará (UFC) - Fortaleza, CE - Brasil

edgar@virtual.ufc.br, rossana@lia.ufc.br, windson@virtual.ufc.br

\begin{abstract}
Field classes favor an approach at the same time more complex and less abstract about the phenomena studied in different domains of knowledge. By adding mobile and ubiquitous technologies we obtain the Ubiquitous Field Classes, which have benefits when compared to traditional. This study presents a systematic mapping with 63 papers on the use of mobile and ubiquitous technologies in education, published between the years 2010 and 2014. Among the main results, we found that most applications is focused on the natural sciences in basic education, it uses the Android platform and is location-aware. From this survey, we identified a number of important requirements for ubiquitous field classes. For demonstration purposes, we present two applications that show the requirements identified.
\end{abstract}

Resumo. As aulas de campo favorecem uma abordagem ao mesmo tempo mais complexa e menos abstrata dos fenômenos estudados em diferentes domínios do conhecimento. Ao incorporar as tecnologias móveis e ubíquas temos as Aulas de Campo Ubíquas, que apresentam beneficios quando comparadas às tradicionais. Este estudo apresenta um mapeamento sistemático elaborado a partir da análise de 63 artigos com experiências sobre o uso das tecnologias móveis e ubíquas na educação, publicados entre os anos de 2010 e 2014. Dentre os principais resultados, constatou-se que a maioria das aplicações é voltada para as ciências naturais no ensino básico, utiliza a plataforma Android e é sensível à localização do aluno. A partir desse levantamento, foi identificado um conjunto de requisitos importantes para as aulas de campo ubíquas. Para fins de demonstração, são apresentadas duas aplicações que mostram o funcionamento de forma integrada dos requisitos identificados.

\section{Introdução}

As aulas de campo possibilitam uma abordagem ao mesmo tempo mais complexa e menos abstrata dos fenômenos estudados em diferentes domínios do conhecimento [Roslin et al., 2013]. Elas também promovem o espírito científico dos alunos por meio do aumento da capacidade de observação e descoberta em diversas áreas, que vão desde matérias do ensino fundamental até disciplinas do ensino superior.

Nesse trabalho, utilizaremos o termo Aulas de Campo Ubíquas (UFC Ubiquitous Field Classes) para referenciar as práticas educativas realizadas em campo com suporte das tecnologias móveis e ubíquas. Diversas pesquisas têm demonstrado os benefícios do uso das tecnologias móveis e ubíquas em aulas em campo [Marçal et al., 2013; Wu et al., 2013; Lo e Quintana, 2013]. Dentre os quais se destacam: identificação 
do contexto do aluno, através de sensores, para entrega de conteúdos e exercícios de acordo com a situação dele naquele momento; registro das preferências dos estudantes para recomendações futuras; e, comunicação entre alunos e professores.

Os sistemas móveis e ubíquos aplicados a aulas de campo podem apresentar variedade em termos de: área de ensino, como ciências naturais ou aprendizagem de idiomas; nível de escolaridade, como ensino básico ou superior; mecanismo de sensoriamento, como GPS ou bússola; tecnologia de transmissão de dados sem fio, como consulta online ou envio de arquivos via email; sistema operacional do dispositivo móvel, como Android ou iOS; entre outros. Desta forma, os profissionais que vão construir ou utilizar esses softwares precisam de informações que fundamentem a escolha das opções mais adequadas às suas realidades.

Este artigo apresenta um mapeamento sistemático realizado a partir de trabalhos publicados entre os anos de 2010 e 2014 com experiências de aulas de campo ubíquas. Pretende-se apresentar um retrato com a visão geral dos sistemas educativos móveis e ubíquos utilizados em campo. Além disso, com base nas práticas observadas nesse levantamento, foi identificado um conjunto de requisitos importantes para servir como referência para o desenvolvimento ou a escolha de sistemas móveis e ubíquos para aulas de campo. Por fim, são apresentadas duas aplicações que demonstram o funcionamento dos requisitos identificados de forma integrada em aulas de campo ubíquas.

\section{Mapeamento Sistemático}

\subsection{Metodologia do Mapeamento}

Para a realização deste mapeamento sistemático, foi adotado o processo de revisão similar ao apresentado em [Borges et al., 2013]. Este processo baseia-se nas questões de pesquisas e em triagens dos artigos, a partir da string de busca e aplicação de critérios sobre o título, o resumo e o artigo em si. As questões de pesquisa utilizadas foram:

- Q1 - Quais as áreas de conhecimento que estão utilizando as tecnologias ubíquas em campo?

- Q2 - Quais os níveis de escolaridade dos alunos que participam das aulas de campo ubíquas?

- Q3 - Quais as atividades de aprendizagem estão sendo realizadas através desses sistemas ubíquos?

- Q4 - Quais informações contextuais estão sendo usadas nesses sistemas?

- Q5 - Em quais Sistemas Operacionais estão funcionando as aulas de campo ubíquas?

- Q6 - Quais as formas de transmissão de dados estão sendo utilizadas por essas aplicações?

Foram realizados alguns testes com diferentes combinações de palavras para definir como seria a busca. Optou-se por utilizar uma string de busca mais abrangente e aplicar posteriormente critérios de exclusão, para recuperar um número maior de artigos e minimizar as possibilidades de eliminar algum trabalho relevante. A string de busca utilizada foi "ubiquitous learning" OR "pervasive learning". Tendo em vista o

\footnotetext{
${ }^{1}$ Ubiquitous Learning e Pervasive Learning são termos que referenciam o uso dos recursos da computação ubíqua e da computação pervasiva na educação e são muitas vezes usados como sinônimos.
} 
surgimento frequente de novas tecnologias móveis e ubíquas, decidiu-se por considerar apenas os trabalhos dos últimos cinco anos (entre 2010 e 2014). Então, em resumo: primeiramente foram incluídos todos os artigos nesse período que atendessem a string de busca e, posteriormente, foram aplicados os filtros (critérios de exclusão).

Através dos critérios de exclusão, buscou-se deixar apenas os artigos que apresentavam sistemas móveis e ubíquos voltados para o ensino e a aprendizagem com experimentação utilizando usuários reais e em ambientes fora de sala de aula. Além disso, para viabilizar a análise, artigos que não estavam escritos em inglês foram retirados do resultado final. A Tabela 1 apresenta as quantidades de artigos que foram identificados inicialmente e que foram sendo triados ao longo do processo do mapeamento sistemático, agrupados por biblioteca digital.

Tabela 1. Quantidade de artigos resultantes do mapeamento sistemático.

\begin{tabular}{|c|c|c|c|}
\hline Biblioteca Digital & Após a busca inicial & Após $1^{\circ}$ Filtro & Após $2^{\circ}$ Filtro \\
\hline $\mathrm{ACM}^{2}$ & 151 & 32 & 11 \\
\hline IEEE Xplore $^{3}$ & 187 & 60 & 24 \\
\hline Science Direct ${ }^{4}$ & 263 & 78 & 28 \\
\hline Totais & 601 & 170 & 63 \\
\hline
\end{tabular}

\subsection{Análise dos Resultados}

A partir da análise dos 63 artigos $^{5}$ finais selecionados e considerando as questões de pesquisa propostas, esta seção apresenta os principais resultados desse mapeamento sistemático. A Figura 1 apresenta um mapa mental que mostra uma visão geral do perfil dos sistemas móveis e ubíquos utilizados em aulas de campo. Os dados estão agrupados de acordo com as questões de pesquisa estabelecidas nesse mapeamento.

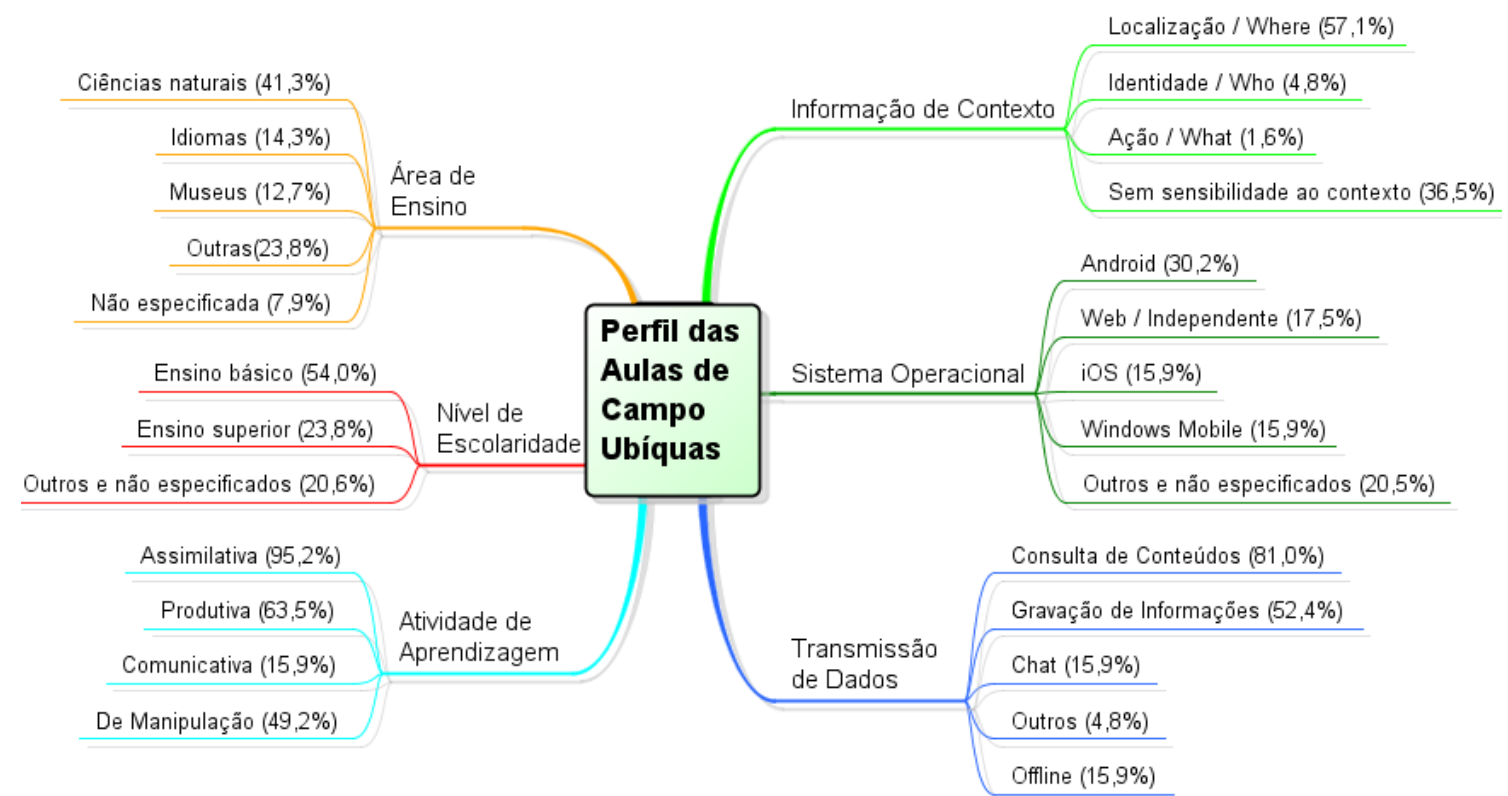

Figura 1. Perfil das Aulas de Campo Ubíquas

\footnotetext{
${ }^{2}$ http://dl.acm.org/

${ }^{3} \mathrm{http}: / /$ ieeexplore.iee.

${ }^{4} \mathrm{http}: / /$ www.sciencedirect.com/

${ }^{5}$ A lista com os 63 artigos está disponível em: https://www.dropbox.com/s/5uben4rqcrfz2e9/listagem_artigos.pdf?dl=0
} 
Sobre a primeira questão de pesquisa (Q1), a partir do mapa da Figura 1, observa-se que a área que apresentou a maior quantidade de artigos foi o ensino de ciências naturais (com 41,3\%), que envolviam o estudo, por exemplo, de ecologia e biologia. Outras áreas que aparecem em destaque são o ensino de idiomas e as aulas em museus. Também foram observados artigos com aulas de campo ubíquas em computação e agricultura.

Com relação ao nível de escolaridade dos alunos envolvidos (Q2), os dados obtidos indicam que mais da metade dos estudos selecionados $(54 \%)$ é voltada para jovens do ensino básico. Em 23,8\% dos artigos selecionados, as experiências com aulas de campo ubíquas foram realizadas com alunos universitários.

Para analisar quais eram as principais atividades de aprendizagem utilizadas nas aulas de campo ubíquas (Q3) tomou-se como base o modelo do DialogPlus toolkit [Conole, 2008]. Este apresenta uma taxonomia para auxiliar professores na construção de atividades de aprendizagem suportadas por recursos digitais e estabelece que as tarefas que os alunos podem executar são agrupadas em seis categorias: assimilativas, produtivas, comunicativas, de manipulação, de simulação e experimentais. A Tabela 2 mostra as tarefas que compõem as atividades de aprendizagem identificadas nos artigos selecionados (Q3). Elas estão agrupadas de acordo com a categorização proposta no DialogPlus. Considerando esse agrupamento, constatou-se um predomínio das atividades assimilativas, que estão presentes em quase todos os trabalhos $(95,2 \%)$.

Um dos benefícios do uso das tecnologias da computação ubíqua para o ensino é a possibilidade da identificação do contexto em que o aluno se encontra no momento da prática educativa. Para fazer a análise das informações contextuais foi utilizado como base o modelo dos 5 Ws de Abowd e Mynatt (2000): When (tempo), Why (motivo), Who (identidade), Where (localização) e What (ação). Conforme pode ser observado na Figura 1, a principal informação de contexto utilizada nas aulas de campo ubíquas (Q4) é o local onde o aluno se encontra (Where), presente em 57,1\% dos estudos. Com percentuais bem menores se encontram as informações contextuais de identidade do usuário (Who) e ação realizada (What). Além disso, percebemos que mais de um terço dos sistemas presentes nos artigos pesquisados (36,5\%) não implementam nenhuma forma de sensibilidade ao contexto ${ }^{6}$.

Tabela 2. Tarefas identificadas no mapeamento agrupadas por categorias.

\begin{tabular}{ll|ll}
\hline Categoria & Tarefa & Categoria & Tarefa \\
\hline Assimilativa & Ler textos & Produtiva & Anotar textos \\
\hline & Ver imagens & Gravar áudios \\
\hline & Assistir a vídeos & Gravar vídeos \\
\hline & Escutar áudios & Desenhar na tela \\
\hline & Visualizar Mapas & Responder questionários \\
\hline & & & \\
\hline & Interagir com os alunos & De Manipulação & Coletar a localização \\
\hline & Interagir com o professor & Capturar fotos \\
\hline
\end{tabular}

${ }^{6}$ Consiste na capacidade do sistema em coletar informações contextuais, as quais se referem a todo e qualquer dado que possa ser utilizado para caracterizar uma entidade (pessoa, local ou objeto) relevante para a interação do usuário [Dey e Abowd, 1999]. 
O gráfico da Figura 2.A) mostra a relação entre os sensores adotados e o tipo de informação de contexto utilizada. É importante destacar que na maioria dos estudos, os sensores são utilizados de forma combinada para capturar o contexto do aluno. A partir do gráfico, conclui-se que a câmera digital do dispositivo do aluno foi o sensor mais utilizado, presente em 14 artigos. Em particular, isso ocorreu pelo fato da câmera poder ser usada tanto para captura de imagens quanto para obtenção da localização do aluno através da leitura de QR-codes (códigos de barra Quick Response).

Com relação à quinta questão de pesquisa desse mapeamento (Q5), o sistema operacional (SO) Android se destaca como a plataforma de quase um terço dos sistemas móveis e ubíquos analisados (30,2\%). A segunda plataforma mais utilizada não se trata de um sistema operacional propriamente: $17,5 \%$ dos sistemas funcionam em sites Web, que independem de SO. Em seguida estão os sistemas operacionais iOS e Windows Mobile, ambos presentes em 15,9\% dos artigos selecionados. Em 20,5\% dos trabalhos são utilizados outros sistemas operacionais ou não foram especificados nos artigos.

Além de identificar o Sistema Operacional, buscou-se investigar como o sistema móvel e ubíquo foi desenvolvido. Como se pode observar no gráfico da Figura 2.B), a maioria dos sistemas analisados foi desenvolvida especificamente para aquele estudo mostrado no artigo. Apesar de fornecerem poucas informações sobre os aspectos de implementação, foram identificadas como as principais linguagens de programação utilizadas: Java, Objective C, C\# e HTML5. Além do desenvolvimento específico dos sistemas, os artigos analisados também utilizavam softwares de mercado já existentes ou ferramentas de autoria para construir os aplicativos a serem utilizados no estudo.

A última questão analisada nesse mapeamento foi sobre os tipos de transmissão de dados via Internet que eram realizados nas aulas de campo ubíquas (Q6). Nesse aspecto, destacam-se em primeiro e segundo lugar, respectivamente, a consulta (81\%) e a gravação $(52,4 \%)$ de informações. Os dados sobre essa questão indicam conclusões semelhantes com a Q3 (tipos de atividades de aprendizagem). Em ambas as questões destaca-se a consulta a conteúdos, seguida pelo registro das anotações e, com um percentual bem menor, as atividades de interação entre os participantes da aula. Também é importante destacar que em $15,9 \%$ dos estudos os sistemas não realizavam nenhum tipo de transmissão de dados em campo. Enquadra-se nesse caso, por exemplo, aquela aplicação na qual o conteúdo já está inserido no seu próprio banco de dados.

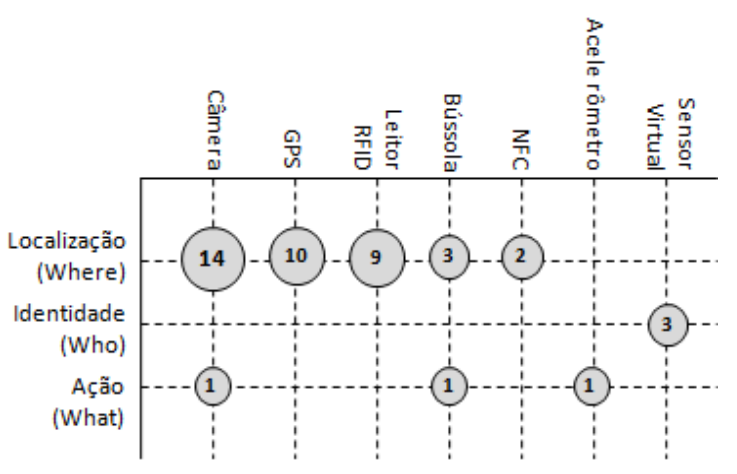

A)

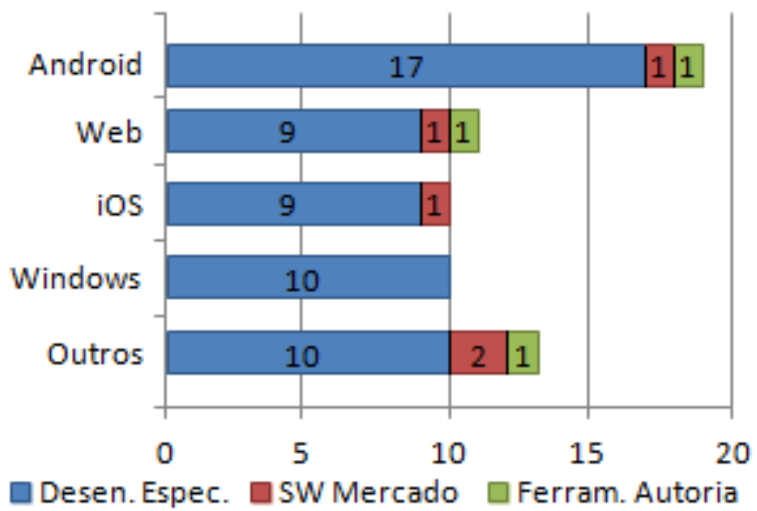

B)

Figura 2. A) Comparativo entre os sensores e as informações contextuais; B)Comparativo entre os sistemas operacionais e a abordagem de desenvolvimento. 


\section{Requisitos}

A partir do mapeamento sistemático descrito na seção anterior e das experiências analisadas, esta seção apresenta um conjunto de requisitos identificados como importantes em aulas de campo ubíquas. Com isso, pretende-se estabelecer um guia para suporte ao desenvolvimento de novos sistemas móveis e ubíquos para aulas de campo ou para seleção entre softwares existentes. A Tabela 3 apresenta os requisitos funcionais e não-funcionais identificados.

Tabela 3. Requisitos funcionais e não-funcionais de aulas de campo ubíquas.

\begin{tabular}{cl}
\hline \multicolumn{2}{c}{ Requisitos de Aulas de Campo Ubíquas } \\
\hline Não-Funcionais & Funcionais \\
\hline $\begin{array}{l}\text { Sensibilidade ao contexto do } \\
\text { aluno }\end{array}$ & $\begin{array}{l}\text { Disponibilizar o conteúdo em diferentes } \\
\text { mídias }\end{array}$ \\
\hline Interface multimodal & $\begin{array}{l}\text { Registrar o material produzido pelo } \\
\text { aluno }\end{array}$ \\
\hline Compatibilidade com & $\begin{array}{l}\text { Possibilitar interação sem fio entre } \\
\text { alunos e professores }\end{array}$ \\
\hline dispositivos móveis & $\begin{array}{l}\text { Consultar material didático em servidor } \\
\text { remoto }\end{array}$ \\
\hline & $\begin{array}{l}\text { Armazenar as informações registradas } \\
\text { em servidor centralizado }\end{array}$ \\
\hline
\end{tabular}

\subsection{Requisitos Não-Funcionais}

Enquanto executam as atividades em campo, diferenças de conhecimento e comportamento são percebidas entre os estudantes. Neste momento, ferramentas de computação ubíqua podem ampliar os benefícios da aula de campo ao considerar o contexto no qual o aluno se encontra. A maioria dos estudos analisados $(63,5 \%)$ nesse mapeamento utiliza alguma informação de contexto durante as atividades de aprendizagem na aula de campo.

Conforme [Oviatt, 2003], os sistemas com interface multimodal são aqueles que processam duas ou mais formas de entrada do usuário de maneira coordenada, por exemplo, via voz ou toque na tela ou gestos. Esses recursos tornam-se ser importantes durante as aulas de campo, quando os alunos precisam executar diferentes tarefas, como observar o ambiente, escutar as orientações do professor e fazer várias anotações. Dentre os trabalhos selecionados, diferentes formas de entrada foram utilizadas para capturar a interação do aluno com o sistema, como o microfone e a câmera.

Em todos os estudos analisados no mapeamento sistemático, o aluno utiliza um smartphone ou um tablet para acessar os recursos do sistema móvel e ubíquo. Portanto, tal dispositivo torna-se essencial para viabilizar as aulas de campo ubíquas, independentemente do sistema operacional utilizado. O mapeamento ainda indica que a aplicação pode ser específica para um determinado SO ou independente de plataforma ao funcionar na Web.

\subsection{Requisitos Funcionais}

Uma das premissas da aprendizagem multimídia [Mayer, 2005] é que os seres humanos possuem canais diferentes para o processamento das informações visuais e auditivas 
(canal dual). Assim, um ambiente multimídia para aprendizagem precisa apresentar conteúdo utilizando diferentes mídias que promovam estímulos visuais e auditivos para que os alunos possam construir representações mentais sobre o que está sendo estudado.

Muitos dos trabalhos pesquisados apresentam sistemas móveis e ubíquos que combinam diferentes mídias para apresentação dos conteúdos. Em geral, ocorre a associação de textos com imagens. Porém, áudios e vídeos também são utilizados, seja para gravação ou visualização.

Conforme indicado nesse mapeamento, mais da metade dos artigos selecionados $(63,5 \%)$ apresentam algum tipo de atividade produtiva. Ou seja, os alunos anotam (via texto ou áudio ou vídeo ou outra forma de entrada) informações importantes para aprendizagem deles, que precisam ser armazenadas pelo sistema. Tendo em vista a variedade e a importância das informações obtidas em campo, é necessário que todos os dados sejam armazenados de forma integrada.

Durante a aula de campo, em diversos momentos os alunos estão dispersos geograficamente e precisam esclarecer dúvidas com o professor ou trocar informações com os colegas. Assim, torna-se um requisito importante para aulas de campo ubíquas a possibilidade dos participantes se comunicarem utilizando as tecnologias sem fio.

Os números apresentados nesse mapeamento demonstram que quase todas as aplicações $(95,2 \%)$ apresentavam algum tipo de atividade assimilativa. Em algumas dessas atividades de aprendizagem, o conteúdo necessário não estava disponível no dispositivo móvel do aluno. Nesse momento, torna-se importante que o sistema possa acessar a um servidor remoto para consultar informações complementares. Esta funcionalidade amplia as possibilidades do aluno durante as aulas de campo, dando-lhe mais autonomia durante a prática.

Um dos principais benefícios da aula de campo é possibilitar que as anotações feitas em campo possam ser analisadas posteriormente [Roslin et al., 2013]. Desta forma, além de poder registrar as informações de forma integrada é importante que esses dados sejam persistidos em um servidor centralizado, para consultas posteriores.

Sobre esse requisito, duas estratégias se destacam nos artigos analisados: o registro dos dados online no servidor durante a aula de campo; e o armazenamento dos dados localmente no dispositivo móvel para posterior exportação das informações. A escolha da opção mais apropriada passa pela análise da disponibilidade da Internet em campo e da capacidade de armazenamento do dispositivo móvel do aluno.

\section{Exemplos de Implementação dos Requisitos}

Para fins de demonstração, são apresentadas duas aplicações que implementam os requisitos identificados na seção anterior: Geomóvel [Marçal et al., 2013] e CAULS [Chen e Huang, 2012]. O primeiro trata-se de um software resultado de pesquisas do nosso grupo iniciadas em 2011 [Viana et al., 2011] com tecnologias móveis e ubíquas no ensino de geologia. O segundo foi escolhido considerando público e área de ensino diferentes do Geomóvel, para ampliar o alcance da demonstração proposta nessa seção. Tomando-se como base as características de uma aula de campo ubíqua da Figura 1, a Tabela 4 e a Tabela 5 apresentam respectivamente os perfis do Geomóvel e do CAULS. 
Tabela 4. Perfil da aplicação Geomóvel.

\begin{tabular}{|c|c|}
\hline $\begin{array}{c}\text { Característica } \\
\text { Descrição }\end{array}$ & $\begin{array}{c}\text { Característica } \\
\text { Descrição }\end{array}$ \\
\hline Área de Ensino: Geologia & \multirow{4}{*}{$\begin{array}{l}\text { Transmissão de dados através de: } \\
\text { Envio dos dados coletados por email; } \\
\text { Troca de imagens via Bluetooth. }\end{array}$} \\
\hline Nível de Escolaridade: Ensino Superior & \\
\hline Informações de Contexto: Localização (via GPS) & \\
\hline Sistema Operacional: Android & \\
\hline $\begin{array}{l}\text { Atividades de Aprendizagem: } \\
\text { Produtiva. Ex: Registro dos dados do afloram } \\
\text { Assimilativa. Ex: Consulta a instruções sobre } \\
\text { Comunicativa. Ex: Troca de imagens capturad } \\
\text { De Manipulação. Ex: obtenção das coordenada }\end{array}$ & $\begin{array}{l}\text { o (ponto de estudo); } \\
\text { edições geológicas; } \\
\text { entre os alunos; } \\
\text { geológicas. }\end{array}$ \\
\hline
\end{tabular}

O Geomóvel [Marçal et al., 2013] trata-se de um aplicativo Android voltado para auxiliar os alunos do curso de graduação de Geologia em aulas de campo. Dos oito requisitos apontados na seção anterior, apenas a consulta a dados em um servidor não foi implementada no Geomóvel, devido à impossibilidade de conexão com a Internet na maioria das aulas de campo de geologia. Nesse caso, foi usada a base de dados do próprio aplicativo para armazenar o conteúdo necessário.

Tabela 5. Perfil da aplicação CAULS.

\begin{tabular}{|c|c|}
\hline $\begin{array}{c}\text { Característica } \\
\text { Descrição }\end{array}$ & $\begin{array}{c}\text { Característica } \\
\text { Descrição }\end{array}$ \\
\hline Área de Ensino: História & \multirow{4}{*}{$\begin{array}{l}\text { Transmissão de dados através de: } \\
\text { Consulta de informações no servidor; } \\
\text { Registro da localização no servidor; } \\
\text { Gravação de avaliações no servidor. }\end{array}$} \\
\hline Nível de Escolaridade: Ensino Fundamental & \\
\hline Informações de Contexto: Localização (via RFID ${ }^{7}$ ) & \\
\hline Sistema Operacional: Windows Mobile & \\
\hline
\end{tabular}

Atividades de Aprendizagem:

Produtiva. Ex: Gravação das respostas a questionários de avaliação no servidor;

Assimilativa. Ex: Consulta de informações sobre os artefatos aborígenes no servidor;

De Manipulação. Ex: obtenção da localização do aluno através das etiquetas RFID.

O artigo [Chen e Huang, 2012] apresenta o CAULS (context-aware ubiquitous learning system), um sistema para alunos do ensino fundamental utilizarem durante visitas a museus, com um estudo de caso em história aborígene de Taiwan. O único requisito não implementado pelo CAULS, dentre os identificados na seção anterior, é a possibilidade de interação entre os participantes da aula no museu através do sistema.

\section{Trabalhos Relacionados}

Em [Hwang et al., 2008], os autores apresentam requisitos, estratégias e questões importantes que devem ser levadas em consideração na construção de sistemas ubíquos educativos sensíveis ao contexto. Os pesquisadores focam na questão da sensibilidade ao contexto, afirmando que os sistemas devem se adaptar ao usuário, fornecer conteúdo personalizado, serem proativos e funcionar em diferentes dispositivos móveis.

\footnotetext{
${ }^{7}$ RFID é um método de identificação automática por radiofrequência através de leitores e etiquetas no padrão RFID ("RadioFrequency IDentification").
} 
O estudo mostrado em [Wu et al., 2012] faz um mapeamento sistemático com pesquisas sobre mobile learning entre os anos de 2003 e 2010. Dentre as principais constatações, os autores destacam que o foco principal dos artigos selecionados era avaliar o uso das tecnologias móveis na aprendizagem. Eles também afirmam que a maioria $(86 \%)$ demonstra que o uso das tecnologias móveis favorece a aprendizagem.

A pesquisa mostrada em [Shuib et al., 2015] analisou cinquenta e cinco artigos que empregaram dispositivos móveis na educação. A principal conclusão dos autores foi o fato da maioria dos trabalhos indicarem que as tecnologias móveis promovem um impacto positivo no aprendizado dos alunos. Além de destacar os benefícios, os autores apontam preocupações importantes insegurança e limitações dos dispositivos móveis.

As constatações fornecidas pelos trabalhos relacionados são informações relevantes, porém diferem deste artigo porque fazem uma avaliação mais abrangente sobre as vantagens e desvantagens dos sistemas móveis e ubíquos na educação. Nosso trabalho foca no desenvolvimento desse tipo de software e busca, de forma específica, identificar que requisitos são importantes e como eles podem ser implementados.

\section{Conclusão}

Diversos pesquisadores têm conduzido experiências que demonstram os efeitos positivos do uso das tecnologias móveis e ubíquas aplicadas em aulas de campo. A partir de um mapeamento sistemático sobre essas pesquisas, identificou-se um conjunto de requisitos funcionais e não funcionais importantes para aulas de campo ubíquas. Para a elaboração dessa lista, foi realizado um levantamento com artigos na área publicados entre os anos de 2010 e 2014. Buscou-se, com esse estudo, definir o perfil dos sistemas móveis e ubíquos utilizados em aulas de campo.

A título de demonstração, foram apresentados os perfis de dois diferentes sistemas móveis e ubíquos para apoio a aulas de campo. Com isso, foi possível mostrar duas possibilidades distintas de implementação dos requisitos identificados. Além disso, outra contribuição desse trabalho é servir como referência para a descoberta de oportunidades para novas pesquisas com sistemas móveis e ubíquos na educação, por exemplo: através da identificação de áreas pouco investigadas, como o uso das ferramentas de interação entre os participantes em aulas de campo ubíquas.

Como trabalho futuro, pretende-se elaborar uma linguagem de modelagem que possibilite a especificação de sistemas móveis e ubíquos considerando os requisitos identificados. Assim, espera-se estabelecer um modelo genérico que possa ser usado para representar aulas de campo ubíquas de diferentes áreas do conhecimento.

\section{Referências}

Abowd, G. D., e Mynatt, E. D. (2000). Charting past, present, and future research in ubiquitous computing. ACM Transactions on Computer-Human Interaction (TOCHI), 7(1), 29-58

Borges, S. D. S., Reis, H. M., Durelli, V. H., Bittencourt, I. I., Jaques, P. A., \& Isotani, S. (2013). Gamificação Aplicada à Educação: Um Mapeamento Sistemático. In XXIV Simpósio Brasileiro de Informática na Educação. 
Chen, C. C., \& Huang, T. C. (2012). Learning in a u-Museum: Developing a contextaware ubiquitous learning environment. Computers \& Education, 59(3), 873-883.

Conole, G. (2008). The role of mediating artefacts in learning design. Handbook of research on learning design and learning objects: Issues, applications and technologies, 187-207.

Dey, A. K. e Abowd, G. D. (1999). Towards a better understanding of context and context-awareness. In: GELLERSEN, H.-W. (Ed.). Handheld and Ubiquitous Computing. [S.1.]: Springer Berlin Heidelberg, 1999, p. 304-307.

Hwang, G. J., Tsai, C. C., \& Yang, S. J. (2008). Criteria, Strategies and Research Issues of Context-Aware Ubiquitous Learning. Educational Technology \& Society, 11(2), 81-91.

Lo, W. T., e Quintana, C. (2013). Students' use of mobile technology to collect data in guided inquiry on field trips. In Proceedings of the 12th International Conference on Interaction Design and Children (pp. 297-300). ACM.

Marçal, E., Andrade, R. M., Viana, W., Rodrigues, D., Maia, M., Mendes, W., ... \& Benicio, W. (2013). Geomóvel: Um Aplicativo para Auxílio a Aulas de Campo de Geologia. In Anais do Simpósio Brasileiro de Informática na Educação.

Mayer, R. E. (2005). Cognitive theory of multimedia learning. In R. Mayer (Ed.), Cambridge handbook of multimedia learning (pp. 31-48). New York: Cambridge University Press.

Oviatt, S. (2003). Multimodal interfaces. The human-computer interaction handbook: Fundamentals, evolving technologies and emerging applications, 286-304.

Roslin, Y. A., Maga, J. P., Rosales, A. B., Cereno, R., \& Tapay, N. E. (2013). Social impact of ecotourism on the behavior of students on educational field trips to Makiling Botanic Gardens in the University of the Philippines Los Baños. USM R\&D Journal, 17(1).

Shuib, L., Shamshirband, S., \& Ismail, M. H. (2015). A review of mobile pervasive learning: Applications and issues. Computers in Human Behavior, 46, 239-244.

Viana, W., Maia, M., Andrade, R., de Lima, J. V., Junior, J. C. F., Marcal, E. e Sanchez, J. (2011). Towards context-aware and mobile e-learning applications. In: TISE 2011, Congreso Internacional de Informática Educativa, 2011, Santiago, Chile.

Wu, P. H., Hwang, G. J., \& Tsai, W. H. (2013). An Expert System-based ContextAware Ubiquitous Learning Approach for Conducting Science Learning Activities. Educational Technology \& Society, 16(4), 217-230.

Wu, W. H., Wu, Y. C. J., Chen, C. Y., Kao, H. Y., Lin, C. H., \& Huang, S. H. (2012). Review of trends from mobile learning studies: A meta-analysis. Computers \& Education, 59(2), 817-827. 